\title{
ON RECURRENT RANDOM WALKS ON SEMIGROUPS
}

\author{
BY \\ T. C. SUN,(1) A. MUKHERJEA AND N. A. TSERPES(2)
}

\begin{abstract}
Let $\mu$ be a regular Borel probability measure on a locally compact semigroup $S$ and consider the right (resp. left) random walk on $D=\overline{U F^{\pi}}, F=\operatorname{Supp} \mu$, with transition function $P^{n}(x, B) \equiv \mu^{n}\left(x^{-1} B\right)$ (resp. $\mu^{n}\left(B x^{-1}\right)$ ). These Markov chains can be represented as $Z_{n}=X_{1} X_{2} \cdots X_{n}$ (resp. $S_{n}=X_{n} X_{n-1} \cdots X_{1}$ ), $X_{i}$ 's independent $\mu$-distributed with values in $S$ defined on an infinite-sequence space $\left(\Pi_{1}^{\infty} S_{i}, P\right), S_{i}=S$ for all $i$. Let $R_{r}$ (resp. $\left.R_{t}\right)=\left\{x \in D ; P_{x}\left(Z_{n}\left(S_{n}\right) \in N_{x}\right.\right.$ i.o. $)=1$ for all neighborhoods $N_{x}$ of $\left.x\right\}$ and $R_{r}^{\prime}\left(R_{\prime}^{\prime}\right)=\left\{x \in D ; P\left(Z_{n}\left(S_{n}\right) \in N_{x}\right.\right.$ i.o. $)=1$ for all $N_{x}$ of $\left.x\right\}$. Let $S$ be completely simple ( $=E \times G \times F$, usual Rees product) in the results (1), (2), (3), (4), (5) below: (1) $x \in R$, iff $\Sigma \mu^{n}\left(x^{-1} N_{x}\right)=\infty$ for all neighborhoods $N_{x}$ of $x$ iff $\sum \mu^{n}\left(N_{x}\right)=\infty$ for all $N_{x}$ of $x$. (2) Either $R_{r}=R_{l}=\varnothing$ or $R_{r}=R_{l}=D=$ also completely simple. (3) If the group factor $G$ is compact, then there are recurrent values and we have $R_{r}=R_{l}=D=$ completely simple. (4) $R_{r}^{\prime} \neq \varnothing$ implies $R_{r}^{\prime}=R_{r}=R_{l}=D=$ a right subgroup of $S$ (but $R_{\prime}^{\prime}$ may be $\varnothing$ ). (5) $S$ can support a recurrent random walk (i.e., a r. walk with $R_{r} \neq \varnothing$ ) iff $G$ (= the group factor) can support a recurrent random walk. Finally (6) if $S$ is compact abelian, then always $R^{\prime}=R=K=$ the kernel of $S$. These results extend previously known results of Chung and Fuchs and Loynes.
\end{abstract}

1. Introduction. In this paper we introduce the notion of a recurrent random walk on a topological semigroup and establish results parallel to those given in [3] and [6]. In $\$ 2$ we find that the definition of recurrence used in [3] and [6] is not suitable for semigroups. In \$3, using a modified definition, we deal with random walks on locally compact completely simple semigroups which have been shown to be important in the study of random walks on semigroups [7], [8], [9], [10]. The problem of recurrence on general compact semigroups is considered in [11]. A few remarks are mentioned in $\$ 4$ where general compact semigroups are also considered. Some definitions and notations used later on are given in the following paragraphs.

Let $S$ be a locally compact Hausdorff topological semigroup and $\mu$ be a regular Borel probability measure with support $\Phi \subset S$. Let $X_{1}, X_{2}, \ldots$ be a sequence of independent random variables on some probability space $(\Omega, \Sigma, P)$ with values in $S$, having the same distribution.

$$
P(X \in B)=\mu(B), \text { for any Borel } B \subset S .
$$

(As usual, we identify the process $X_{n}$ with the usual coordinate representation

Received by the editors May 24, 1972.

AMS (MOS) subject classifications (1970). Primary 60G50, 60J5, 43A05.

Key words and phrases. Sums of independent random variables with values in a semigroup, stationary recurrent random walks, completely simple topological semigroups.

(1) Partly supported by a Faculty Research Grant from Wayne State University.

(2) Under a USF Research Council Grant. 
process in the sequence space $S^{\infty} \equiv \Omega$, having the same distribution.) The sequence $Z_{n}=X_{1} X_{2} \cdots X_{n}, n=1,2, \ldots$, is called a right random walk on $S$ generated by $\mu$. (A left random walk is similarly defined.) The set

$$
D=\overline{U_{n=1}^{\infty} \Phi^{n},} \text { the closed subsemigroup generated by } \Phi,
$$

is called the support of the random walk $\left\{Z_{n}\right\}$. By the kernel $K$ of $D$ we shall mean the minimal (if it exists) two-sided ideal of $D$. We shall use the following notation:

$$
\begin{aligned}
& A^{-1} B \equiv\{s \in S ; \text { as } \in B \text { for some } a \in A\} \\
& x^{-1} B \equiv\{s \in S ; x s \in B\} . \text { Also, } \\
& Z_{k}^{-1} Z_{n} \equiv X_{k+1} X_{k+2} \cdots X_{n} .
\end{aligned}
$$

We have for Borel $B, P\left(Z_{n} \in B\right)=\mu^{n}(B)$, where $\mu^{n}=\mu * \mu * \cdots * \mu$ (n times). It can be easily verified that $P\left(Z_{n+k} \in B \mid Z_{n}=x\right)=\mu^{k}\left(x^{-1} B\right)=$ the $k$ th transition function for $Z_{n}$. [We assume $Z_{n}$ 's to be measurable.]

The semigroup $S$ is said to be completely simple if $S$ is homeomorphic and isomorphic to a topological product $S \equiv E \times G \times F$ where $E, F$ are left-zero and right-zero semigroups resp., $G$ is a locally compact group and the multiplication of elements in $E \times G \times F$ is defined by

$$
(e, g, f) \cdot\left(e^{\prime}, g^{\prime}, f^{\prime}\right)=\left(e, g\left(f e^{\prime}\right) g^{\prime}, f^{\prime}\right) .
$$

If $\bar{e} \in \mathcal{\varepsilon}(S) \equiv$ the set of idempotent elements of $S$, then in the above representation we may (and do) choose $E \equiv \varepsilon(S \bar{e}), G \equiv \bar{e} S \bar{e}, F \equiv \varepsilon(\bar{e} S)$, so that $F E \subset G$ and the homeomorphism is given by

$$
\begin{aligned}
\eta: E \times G \times F \rightarrow S, & \eta(e, g, f) & =e g f, \\
\eta^{-1}: S \rightarrow E \times G \times F, & \eta^{-1}(s) & =\left(s(\bar{e} s \bar{e})^{-1}, \bar{e} s \bar{e},(\bar{e} s \bar{e})^{-1} s\right)
\end{aligned}
$$

(see [1, pp. 46, 61] and [10]). If $S$ is isomorphic to $G \times F$ where the multiplication of elements in $G \times F$ is reduced to direct-product multiplication $(g, f) \cdot\left(g^{\prime}, f^{\prime}\right)$ $=\left(g g^{\prime}, f^{\prime}\right)$, then $S$ is called a right group. (A left group is similarly defined.) A right group is right simple $(x S=S$ for all $x \in S$ ) and left cancellative and every idempotent is a left identity in $S$. We observe that if $S$ is completely simple and $x=(e, g, f) \in S$, then $x S=\{e\} \times G \times F$ is a right group [1, p. 46].

2. Preliminaries. Suppose $X_{1}, X_{2}, \ldots$ are independent identically distributed random variables with values in a locally compact group $G$. In [3] and [6] an element $g \in G$ is defined to be recurrent for the right random walk $Z_{n}$ $=X_{1} X_{2} \cdots X_{n}$ if

(1) $P\left(Z_{n} \in N_{g}\right.$ infinitely often (i.o.)) $\equiv P\left(\cap_{n=1}^{\infty} \cup_{k=n}^{\infty}\left\{Z_{k} \in N_{g}\right\}\right)=1$, for all open neighborhoods $N_{g}$ of $g$. Let $R^{\prime} \equiv$ the set of recurrent elements in the sense of (1).

An element $g \in G$ is called possible if for each $N_{g}$ there exists a $k$ such that $P\left(Z_{k} \in N_{g}\right)>0$. It was proved that 
(i) either there is no recurrent element or the set of all possible values are recurrent and form a closed subgroup of $G$;

(ii) there are recurrent values if and only if $\Sigma P\left(Z_{n} \in N\right)=\infty$ for all neighborhoods $N$ of the identity of $G$.

The following example shows that condition (1) is not natural for random walks on semigroups.

Example 2.1. Let $S=E \times G$ be a left group where the group $G=\{1, a\}$ and the left-zero semigroup $E=\left\{e_{1}, e_{2}\right\}$ so that $e_{1} e_{2}=e_{1}^{2}=e_{1}, e_{2} e_{1}=e_{2}^{2}=e_{2}$. Let $\mu\{(e, g)\}=\frac{1}{4}$ for each $(e, g) \in S$. Consider the right random walk generated by $\mu, Z_{n}=X_{1} X_{2} \cdots X_{n}$. Since $P\left(Z_{n}=\left(e_{1}, 1\right)\right.$ i.o. $\left.\mid Z_{1}=\left(e_{1}, 1\right)\right)=1$, we should say that $\left(e_{1}, 1\right)$ is recurrent. However, according to $(1),\left(e_{1}, 1\right)$ is not recurrent because $P\left(Z_{n}=\left(e_{1}, 1\right)\right.$ i.o. $)=\frac{1}{2}<1$.

This is due to the fact that a random walk on semigroups may have disjoint nonempty communicating classes;(3) certainly this cannot happen in groups. Also we cannot expect statement (i) above, to be true in semigroups because there exist nonessential( $(3)$ classes; this can be seen in the following example (which shows that not every possible state is recurrent).

Example 2.2. Let $S=[0,1]$ with the Euclidean topology and with the multiplication of real numbers as the binary operation. Let $\mu$ be the Lebesgue measure on $S$. Then, $\mu\left((k, 1] x^{-1}\right) \leq \mu((k, 1])$, for $k>0 ; \mu^{2}((k, 1])=\int_{k}^{1} \mu\left((k, 1] x^{-1}\right) \mu(d x)$ $\leq(1-k)^{2}$, and by induction $\mu^{n}((k, 1]) \leq(1-k)^{n}$, for all $k>0$. Hence $\sum \mu^{n}((k, 1])<\infty$ which implies that $P\left(Z_{n} \in(k, 1]\right.$ i.o. $)=0$. Hence $P\left(Z_{n} \in[0\right.$, $k$ ) i.o.) $=1$ for all $k>0$. Therefore 0 is the only recurrent state for $Z_{n}$, while every $x \in(0,1]$ is possible. It is easy to see that $(0,1)$ and $\{1\}$ are nonessential communicating classes.

In the remaining part of this section, let $R^{\prime}$ be the set of recurrent states of $\left\{Z_{n}\right\}$ as defined by (1). Recall that $D$ is the set of possible states for $Z_{n}$. We shall show below that if $S$ is a completely simple semigroup then either $R^{\prime}=\varnothing$ or $R^{\prime}=D=$ a right subgroup of $S$, thus generalizing a corresponding result in [3] and [6].

Lemma 2.1. Let $S$ be a completely simple semigroup and let $R^{\prime} \neq \varnothing$. Then $D$ is contained in a sub-right-group of $S$.

Proof. Suppose $(e, g, f) \in R^{\prime}$ and $\left(e^{\prime}, g^{\prime}, f^{\prime}\right) \in D$ with $e \neq e^{\prime}$. Then there exist neighborhoods $N_{e}, N_{e^{\prime}}$ of $e$ and $e^{\prime}$ in $E$ respectively such that $N_{e} \cap N_{e^{\prime}}=\varnothing$. Let $N_{g}, N_{g^{\prime}}$ be neighborhoods of $g$ and $g^{\prime}$ in $G$ and $N_{f}$ and $N_{f^{\prime}}$ be neighborhoods in $F$. Since $\left(e^{\prime}, g^{\prime}, f^{\prime}\right) \in D$, we have $\mu^{k}\left(N_{e^{\prime}} \times N_{g^{\prime}} \times N_{f^{\prime}}\right)>0$ for some $k \geq 1$. Also

$$
N_{e} \cap N_{e^{\prime}}=\varnothing \Rightarrow\left(N_{e^{\prime}} \times N_{g^{\prime}} \times N_{f^{\prime}}\right)^{-1}\left(N_{e} \times N_{g} \times N_{f}\right)=\varnothing
$$

(3) These terms are used here in the sense of K. L. Chung, Markov chains with stationary transition probabilities, Springer-Verlag, Berlin, 1960, p. 11. 
It follows that

$$
P\left(Z_{n} \in N_{e} \times N_{g} \times N_{f} \text { f.o. (finitely often)) } \geq \mu^{k}\left(N_{e} \times N_{g^{\prime}} \times N_{f^{\prime}}\right)>0 .\right.
$$

Therefore $(e, g, f) \notin R^{\prime}$, a contradiction. Hence there does not exist in $D$ a state $\left(e^{\prime}, g^{\prime}, f^{\prime}\right)$ with $e \neq e^{\prime}$, i.e., $D \subset\{e\} \times G \times F$, a right group. Q.E.D.

Theorem 2.1. If $S$ is completely simple, then either $R^{\prime}=\varnothing$ or $R^{\prime}=D=a$ topological right group in $S$.

Proof. Since $D$ is inside a right group (Lemma 2.1) and since a right group with multiplication from the right is a group, the proof given in [3] and [6] can be applied in our case. In fact one may assume that $D$ itself is a right group by Lemma 2.1 ; if $R^{\prime} \neq \varnothing$, then every $d \in D$ has the property that $\sum \mu^{n}\left(N_{d}\right)=\infty$ for all $N_{d}$ since the set of points with this property is an ideal and $D$ is simple; then using similar arguments as in [6], it follows that if $\left(g, e_{0}\right) \in R^{\prime}$ and $(c, e) \in D$, then $\left(c^{-1} g, e_{0}\right) \in R^{\prime}$ and $R^{\prime}$ is a left ideal. Also by the argument in [6, Theorem 4, p. 452], every idempotent $e \in D$ is in $R^{\prime}$, from which one obtains $R^{\prime}=$ D. Q.E.D.

Following the arguments in [3] and [6] (see above proof) we can also show the following

Theorem 2.2. If $S$ is a right group, then $R^{\prime} \neq \varnothing$ if and only if $\sum_{n=1}^{\infty} P\left(Z_{n} \in N_{a}\right)$ $=\sum \mu^{n}\left(N_{a}\right)=\infty$ for all neighborhoods $N_{a}$ of some $a \in D$.

3. The main results. In this section we shall assume that $S$ is a locally compact, completely simple semigroup, i.e., $S \equiv E \times G \times F$ as in $\$ 1$. From the results in $\$ 2$, we can see that it is more reasonable to define, for random walks on semigroups, a recurrent state as following:

Definition 3.1. We say that $x \in S$ is a recurrent value for the right random walk $Z_{n}=X_{1} X_{2} \cdots X_{n}$ on $S$ if $x \in D$ and, for each neighborhood $N_{x}$ of $x$,

$$
P\left(Z_{n} \in N_{x} \text { i.o. } \mid Z_{1}=x\right) \equiv P_{x}\left(Z_{n} \in N_{x} \text { i.o. }\right)=1 \text {. }
$$

Notations. We write

(i) $P_{x}(E) \equiv P\left(E \mid X_{1}=x\right)$.

(ii) $R \equiv\{x \in D ; x$ is a recurrent value $\}$.

(iii) $x \rightarrow y$ if $x \in D$ and for each neighborhood $N_{y}$ of $y, P_{x}\left(Z_{n} \in N_{y}\right)>0$ for some $n \geq 1$. (Observe that $x \rightarrow y$ if and only if $y \in \overline{x D}=\operatorname{cl}(x D)$ ).)

(iv) $x \rightarrow y$ i.o. if $x \in D$ and for each $N_{y}, P_{x}\left(Z_{n} \in N_{y}\right.$ i.o. $)=1$. (Observe that $x \rightarrow y$ i.o. $\Rightarrow y \in \overline{x D} \Rightarrow x \rightarrow y$.)

The following proposition of independent interest shows that for fixed elements $f, f^{\prime} \in F$, whether $x \rightarrow y$ i.o. is independent of $e \in E$ and depends only on the product of the $G$-coordinates.

Proposition 3.1. Let $x \rightarrow y$ i.o., in which case $y \in \overline{x D}$ and $x, y$ must be of the form $x=\left(e, g_{x}, f\right), y=\left(e, g_{y}, f^{\prime}\right)$ for some $g_{x}, g_{y} \in G$ and some $f, f^{\prime} \in F$. Then 
for any $z, w \in D$ of the form $z=\left(e^{\prime}, g_{z}, f\right), w=\left(e^{\prime}, g_{w}, f^{\prime}\right), z \rightarrow w$ i.o. provided $g_{z}^{-1} g_{w}=g_{x}^{-1} g_{y}$.

Proof. Let $N_{y}=N_{e} \times N_{g,} \times N_{f^{\prime}}$, where $N_{e}, N_{f}$ are neighborhoods of $e$ and $f^{\prime}$ in $E$ and $F$ respectively and $N_{g} \equiv g_{y} N_{u}, N_{u}$ being a neighborhood of the unit element $u$ in the group $G$. We note that

$$
1=P_{x}\left(Z_{n} \in N_{y} \text { i.o. }\right)=P\left(x Z_{n} \in N_{y} \text { i.o. }\right)=P\left(Z_{n} \in x^{-1} N_{y} \text { i.o. }\right)
$$

and

$$
\begin{aligned}
x^{-1} N_{y} & =\left\{(a, b, c) \in S ;\left(e, g_{x}, f\right) \cdot(a, b, c) \in N_{y}\right\} \\
& =\left\{(a, b, c) \in S ;\left(e, g_{x}(f a) b, c\right) \in N_{e} \times g_{y} N_{u} \times N_{f^{\prime}}\right\} \\
& =\left\{(a, b, c) \in S ; c \in N_{f^{\prime}} \text { and } b \in(f a)^{-1} g_{x}^{-1} g_{y} N_{u}\right\}
\end{aligned}
$$

which, given $f, N_{f}$ and $N_{u}$, depends only on $g_{x}^{-1} g_{y}$. Hence, for $N_{w}=N_{e} \times g_{w} N_{u}$ $\times N_{f}$, we have by a computation of $z^{-1} N_{w}$ as in (2), $x^{-1} N_{y}=z^{-1} N_{w}$ as long as $g_{x}^{-1} g_{y}=g_{z}^{-1} g_{w}$. Therefore

$$
P_{z}\left(Z_{n} \in N_{w} \text { i.o. }\right)=P\left(Z_{n} \in z^{-1} N_{w} \text { i.o. }\right)=P\left(Z_{n} \in x^{-1} N_{y} \text { i.o. }\right)=1 .
$$

Q.E.D.

Lemma 3.1. Let $E$ be compact. Then for each $N_{u}$ of the unit element $u$ in $G$ and for each $f \in F$ there exists $N_{f}$ of $f$ in $F$ such that $N_{f} e \subset N_{u}(f e)$ for all $e \in E$.

Proof. Let $N \equiv N_{u}$ and $f \in F$ be given. For each $e \in E$, there exist $N_{f e}$ in $G$, $N_{f}$ in $F, N_{e}$ in $E$, such that:

$$
\begin{aligned}
& N_{f e} N_{f e}^{-1} \subset N, N_{f} N_{e} \subset N_{f e}, \text { and hence, for every } e^{\prime} \in N_{e}, \\
& f e^{\prime} \subset N_{f e}, N_{f e}\left(f e^{\prime}\right)^{-1} \subset N, N_{f} N_{e} \subset N_{f e} \subset N\left(f e^{\prime}\right) \text { for all } e^{\prime} \in N_{e} .
\end{aligned}
$$

Since a finite collection of $N_{e}$ 's cover $E$, say $N_{a}, N_{e_{2}}, \ldots, N_{e_{1}}$, then the desired neighborhood is $N_{f}=\cap N_{f}^{(i)}$, where $N_{f}^{(i)}$ is the neighborhood of $f$ in $F$ chosen above relative to $N_{e 1} i=1,2, \ldots, n$. Q.E.D.

Proposition 3.2. If $x \rightarrow y$ i.o. and $x \rightarrow z$, then $z \rightarrow y$ i.o.

Proof. (i) Suppose $z \nrightarrow y$ i.o. Then $P_{z}\left(Z_{n} \in \tilde{N}_{y}\right.$ f.o. $)=P\left(Z_{n} \in z^{-1} \tilde{N}_{y}\right.$ f.o. $)>\varepsilon$ $>0$ for some neighborhood $\tilde{N}_{y}$ of $y$. Choose $H=C \times G \times F$ where $C \subset E$ is compact such that $P\left(Z_{1} \in H^{c}\right)<\varepsilon / 2$. This implies that $P\left(Z_{n} \in H^{c}\right.$ i.o. $)<\varepsilon / 2$, since the set $\left\{Z_{n} \in H^{c}\right.$ i.o. $\}$ is contained in $\left\{Z_{1} \in H^{c}\right\}$.

(ii) Suppose $x=\left(e, g_{x}, f_{x}\right), y=\left(e, g_{y}, f_{y}\right), z=\left(e, g_{z}, f_{z}\right)$, and $\tilde{N}_{y}=N_{e} \times \tilde{N}_{8}$, $\times \hat{N}_{f,}, N_{y}=N_{e} \times N_{g y} \times N_{f y}, N_{z}=N_{e} \times N_{g z} \times N_{f_{z}}$, where $N_{e}, N_{f_{y}}, \hat{N}_{f_{y}}$, and $N_{f_{z}}$ are neighborhoods of $e, f_{y}$ and $f_{z}$ in $E$ and $F$ respectively, and $N_{g y}=g_{y} N_{u}, \tilde{N}_{g_{y}}=g_{y} \tilde{N}_{u}$ and $N_{g_{z}}=g_{z} \hat{N}_{u}$ with $N_{u}, \hat{N}_{u}, \hat{N}_{u}$ being neighborhoods of the identity $u$ of $G$. It is easy to compute 


$$
\begin{aligned}
& z^{-1} \tilde{N}_{y}=\left\{(a, b, c) \in S ; c \in \tilde{N}_{f y} \text { and } b \in\left(f_{z} a\right)^{-1} g_{z}^{-1} g_{y} \tilde{N}_{u}\right\} \\
& N_{z}^{-1} N_{y}=\left\{(a, b, c) \in S ; c \in N_{f y} \text { and } b \in\left(N_{f_{z}} a\right)^{-1} \hat{N}_{u}^{-1} g_{z}^{-1} g_{y} N_{u}\right\}
\end{aligned}
$$

(iii) Write $\left(z^{-1} \tilde{N}_{y}\right) \cap H=A,\left(N_{z}^{-1} N_{y}\right) \cap H=B$. Since $C$ is compact, by (4) and (5) and Lemma 3.1, for given $z$ and $\tilde{N}_{y}$, we can find $N_{f y}, N_{f_{z}}, \hat{N}_{u}$ and $N_{u}$ such that $B \subset A$.

(iv) Since $x \rightarrow z$, there exists a $k>0$ such that $P_{x}\left(Z_{k} \in N_{z}\right)=P\left(x Z_{k-1} \in N_{z}\right)$ $>0$. Then

$$
\begin{aligned}
P_{x}\left(Z_{n}\right. & \left.\in N_{y} \text { f.o. }\right)=P\left(x Z_{n} \in N_{y} \text { f.o. }\right) \\
& \geq P\left(x Z_{k-1} \in N_{z} \text { and }\left(x Z_{k-1}\right)^{-1}\left(x Z_{n}\right) \in\left(x Z_{k-1}\right)^{-1} N_{y} \text { f.o. }\right) \\
& \geq P\left(x Z_{k-1} \in N_{z} \text { and } Z_{k}^{-1} Z_{n} \in N_{z}^{-1} N_{y} \text { f.o. }\right) \\
& \geq P\left(x Z_{k-1} \in N_{z}\right) \cdot P\left(Z_{n} \in N_{z}^{-1} N_{y} \text { f.o. }\right) \\
& \geq P\left(x Z_{k-1} \in N_{z}\right)\left[P\left(Z_{n} \in N_{z}^{-1} N_{y} \cap H \text { f.o. }\right)-P\left(Z_{n} \in H^{c} \text { i.o. }\right)\right] \\
& \geq P\left(x Z_{k-1} \in N_{z}\right)\left[P\left(Z_{n} \in B \text { f.o. }\right)-\varepsilon / 2\right] \\
& \geq P\left(x Z_{k-1} \in N_{z}\right)\left[P\left(Z_{n} \in A \text { f.o. }\right)-\varepsilon / 2\right] \\
& \geq P\left(x Z_{k-1} \in N_{z}\right)\left[P\left(Z_{n} \in z^{-1} \tilde{N}_{y} \text { f.o. }\right)-\varepsilon / 2\right]>0
\end{aligned}
$$

This is a contradiction. Q.E.D.

Lemma 3.2. If a subsemigroup $D$ of a completely simple semigroup $S=E \times G$ $\times F$ has a minimal right (or left) ideal (of itself), then $D$ is also completely simple.

Proof. By hypothesis, for some $a \in D, a D$ is right simple and being left cancellative ( $a D \subset a S=$ a right group), $a D$ is a right group and contains an idempotent. By $[4, \mathrm{II}$, p. 88 or I, p. 84], $D$ has a completely simple kernel $K=E^{\prime} \times G^{\prime} \times F^{\prime}, E^{\prime} \subset E, F^{\prime} \subset F, G^{\prime} \subset G$, and $G^{\prime}$ is a group. Let $(e, g, f)$ $\in D$. Then $e \in E^{\prime}, f \in F^{\prime}$ by the ideal property of $K$. Hence fe $\in G^{\prime}$ and so $(f e)^{-1} \in G^{\prime}$ so that $\left(e,(f e)^{-1}, f\right) \in K$ and also $(e, g, f) \cdot\left(e,(f e)^{-1}, f\right)=(e, g, f)$ $\in K$. Hence $K=D$.

Proposition 3.3. If $x \rightarrow y$ i.o., then $x D$ is a closed right group and $D$ is completely simple.

Proof. By Proposition 3.2, $y \in \bigcap_{z \in \overline{x D}} \overline{z D} \equiv I$. We claim that $I$ is a minimal right ideal of $D$ and hence by Lemma 3.2, $D$ is completely simple. We only need to prove that $I$ is right simple, i.e., $s I=I$ for all $s \in I$. Let $s \in I$ and let $w \in s I \subset I \subset \overline{x D} \subset x S=$ a right group. Then $s I$ is a right ideal of $D$ and 
$\overline{s I}=s I$ since the left translations in a right group are closed. Hence $I \subset \overline{w D}$ $\subset s I \subset I$. This completes the proof.

In the representation of $S=E \times G \times F$ we may choose:

$E \equiv \varepsilon(S e) \supset E^{\prime} \equiv \varepsilon(D e)$, where $e \in \varepsilon(D)$

$F \equiv \varepsilon(e S) \supset F^{\prime} \equiv \varepsilon(e D)$

$G \equiv e S e \supset G^{\prime} \equiv e D e$, so that

$D=E^{\prime} \times G^{\prime} \times F^{\prime} \subset S=E \times G \times F$.

This representation will be used in the proof of Theorem 3.2 in the sequel.

Definition 3.2. We write $G_{e f} \equiv\{e\} \times G \times\{f\}=$ a typical maximal subgroup of $S \equiv E \times G \times F$, and $u_{e f} \equiv\left(e,(f e)^{-1}, f\right)=$ the unit of $G_{e f}$

Theorem 3.1. Let $S=E \times G \times F$. Then for each idempotent $u_{e f} \in S, u_{e f} \rightarrow u_{e f}$ i.o. $\Leftrightarrow \sum_{n=1}^{\infty} P_{u_{f f}}\left(Z_{n} \in N\right)=\infty$ for every neighborhood $N$ of $u_{\text {ef. }}$

Proof. The " $\Rightarrow$ " part is trivial by the Borel-Cantelli lemma. We shall prove the " $\Leftarrow$ " part in four steps. $\left({ }^{4}\right)$

Step I. Let $k$ be a fixed positive integer. Let $N$ be a given neighborhood of $u_{e f}$. Since

$$
\infty=\sum_{n=1}^{\infty} P_{u_{f}}\left(Z_{n} \in N\right)=\sum_{j=1}^{k} \sum_{i=0}^{\infty} P_{u_{f f}}\left(Z_{j+i k} \in N\right),
$$

there is a $j_{0}$ such that $1 \leq j_{0} \leq k$ and $\sum_{i=0}^{\infty} P_{u_{f}}\left(Z_{j_{0}+i k} \in N\right)=\infty$. Now

$$
\begin{aligned}
1 & \geq P_{u_{f}}\left(Z_{n} \in N \text { for finitely many } n\right) \\
& \geq \sum_{i=0}^{\infty} P_{u_{e f}}\left(Z_{j_{0}+i k} \in N, Z_{n} \notin N \text { for all } n \geq j_{0}+(i+1) k\right)
\end{aligned}
$$

(these sets are pairwise disjoint)

$$
\begin{aligned}
& \geq \sum_{i=0}^{\infty} P_{u_{e f}}\left(Z_{j_{0}+i k} \in N, Z_{j_{0}+i k}^{-1} Z_{n} \notin N^{-1} N \text { for all } n \geq j_{0}+(i+1) k\right) \\
& =\sum_{i=0}^{\infty} P_{u_{f}}\left(Z_{j_{0}+i k} \in N\right) P\left(Z_{n} \notin N^{-1} N \text { for all } n \geq k\right) .
\end{aligned}
$$

Hence

$$
P\left(Z_{n} \notin N^{-1} N \text { for all } n \geq k\right)=0 .
$$

(This result is true for any arbitrary neighborhood $N^{\prime}$ in $\{e\} \times G \times F$ of $u_{e f}$.)

Step II. We find $N_{0} \subset N$ such that $N_{0}=\left\{(e, b, c) ; c \in N_{f}, b \in N_{u}(c e)^{-1}\right\}$, where $N_{f}$ is a neighborhood of $f$ and $N_{u}$ is a neighborhood of the identity $u$ in the group $G$. Then it can be checked easily that

(4) In Step I, we show $P\left(Z_{n} \notin N^{-1} N\right.$ for all $\left.n \geq k\right)=0$. But transition from $N^{-1} N$ to $u_{e f}^{-1} N$ involves complications and this is demonstrated in Steps II and III. Then Step IV completes the proof. 


$$
u_{e f}^{-1} N_{0}=\left\{\left(e^{\prime}, b, c\right) ; b \in\left(f e^{\prime}\right)^{-1}(f e) N_{u}(c e)^{-1}, c \in N_{f}, e^{\prime} \in E\right\} \text {. }
$$

Let $E_{1}$ be a compact subset of $E$.

We can find neighborhoods $N_{f}^{\prime} \subset N_{f}, N_{u}^{\prime} \subset N_{u}$ of $f$ and $u$ respectively such that

$$
N_{1}=\left\{\left(e^{\prime}, b, c\right) ; c \in N_{f}^{\prime}, b \in N_{u}^{\prime}\left(c e^{\prime}\right)^{-1}, e^{\prime} \in E_{1}\right\} \subset u_{e f}^{-1} N_{0}
$$

(see computation of $u_{e f}^{-1} N_{0}$ above). This is possible because for every $e^{\prime} \in E_{1}$ (which is compact), $(f e)^{-1}\left(f e^{\prime}\right) u\left(f e^{\prime}\right)^{-1}(f e) \subset N_{u},(5)$ so that we can find $N_{f}^{\prime}, N_{u}^{\prime}$ such that for every $e^{\prime} \in E_{1}$ and, for every $c \in N_{f}^{\prime},(f e)^{-1}\left(f e^{\prime}\right) N_{u}^{\prime}\left(c e^{\prime}\right)^{-1}(c e)$ $\subset N_{u}$, or $N_{u}^{\prime}\left(c e^{\prime}\right)^{-1} \subset\left(f e^{\prime}\right)^{-1}(f e) N_{u}(c e)^{-1}$, for every $c \in N_{f}^{\prime}$ and every $e^{\prime} \in E_{1}$.

Now we can find $N_{f}^{\prime \prime}\left(\subset N_{f}^{\prime}\right), N_{u}^{\prime \prime}\left(\subset N_{u}^{\prime}\right)$ such that

$$
N_{2}=\left\{\left(e^{\prime}, b, c\right) ; c \in N_{f}^{\prime \prime}, b \in N_{u}^{\prime \prime}\left(c e^{\prime}\right)^{-1}, e^{\prime} \in E_{1}\right\} \subset N_{1},
$$

and

$$
N_{2}^{-1} N_{2} \cap E_{1} \times G \times F \subset N_{1}
$$

This is possible since

$N_{2}^{-1} N_{2} \cap E_{1} \times G \times F$

$$
\begin{aligned}
& =\left\{\left(e^{\prime}, b, c\right) ;\left(e^{\prime \prime}, b^{\prime}, c^{\prime}\right)\left(e^{\prime}, b, c\right) \in N_{2}, \text { where }\left(e^{\prime \prime}, b^{\prime}, c^{\prime}\right) \in N_{2}, e^{\prime} \in E_{1}\right\} \\
& =\left\{\left(e^{\prime}, b, c\right) ; e^{\prime} \in E_{1}, b^{\prime}\left(c^{\prime} e^{\prime}\right) b \in N_{u}^{\prime \prime}\left(c e^{\prime \prime}\right)^{-1},\right. \\
& \text { where } \left.e^{\prime \prime} \in E_{1}, c \in N_{f}^{\prime \prime}, c^{\prime} \in N_{f}^{\prime \prime}, b^{\prime} \in N_{u}^{\prime \prime}\left(c^{\prime} e^{\prime \prime}\right)^{-1}\right\} \\
& \subset\left\{\left(e^{\prime}, b, c\right) ; e^{\prime} \in E_{1}, b \in\left(c^{\prime} e^{\prime}\right)^{-1}\left(c^{\prime} e^{\prime \prime}\right) N_{u}^{\prime \prime-1} N_{u}^{\prime \prime}\left(c e^{\prime \prime}\right)^{-1},\right.
\end{aligned}
$$

where $\left.c^{\prime} \in N_{f}^{\prime \prime}, e^{\prime \prime} \in E_{1}, c \in N_{f}^{\prime \prime}\right\}$

which can be seen to be a subset of $N_{1}$ by properly choosing $N_{f}^{\prime \prime}$ and $N_{u}$ "since for every $e^{\prime}, e^{\prime \prime} \in E_{1},\left(f e^{\prime}\right)^{-1}\left(f e^{\prime \prime}\right) u\left(f e^{\prime \prime}\right)^{-1}\left(f e^{\prime}\right) \subset N_{u}^{\prime}$, so that there exist $N_{f}^{\prime \prime}, N_{u}^{\prime \prime}$ such that

$$
\left(N_{f}^{\prime \prime} e^{\prime}\right)^{-1}\left(N_{f}^{\prime \prime} e^{\prime \prime}\right) N_{u}^{\prime \prime-1} N_{u}^{\prime \prime}\left(N_{f}^{\prime \prime} e^{\prime \prime}\right)^{-1}\left(N_{f}^{\prime \prime} e^{\prime}\right) \subset N_{u}^{\prime} .
$$

Step III. We claim that for all $k$ (a positive integer)

$$
P_{u_{d}}\left(Z_{n} \notin N \text { for all } n \geq k\right)=0 \text {. }
$$

To prove this, let $\varepsilon>0$. Let $E_{1}$ be a compact subset of $E$ such that $\mu\left(\left(E-E_{1}\right) \times G \times F\right)<\varepsilon$. By Step II, we can find $N_{2}$, a relative neighborhood of $u_{e f}$ in $\{e\} \times G \times F$, such that $N_{2}^{-1} N_{2} \cap E_{1} \times G \times F \subset u_{e f}^{-1} N$. Now,

(s) Note that the mapping $(f, e) \rightarrow f \cdot e$ from $F \times E \rightarrow G$ is continuous by the definition of a completely simple semigroup. 


$$
\begin{aligned}
P_{u_{f}}\left(Z_{n} \notin N \text { all } n \geq k\right) \leq & P\left(Z_{n} \notin u_{e f}^{-1} N \text { all } n \geq k\right) \\
\leq & P\left(Z_{n} \notin N_{2}^{-1} N_{2} \cap E_{1} \times G \times F \text { all } n \geq k\right) \\
\leq & P\left(Z_{n} \notin N_{2}^{-1} N_{2} \text { all } n \geq k\right) \\
& +P\left(Z_{n} \in\left(E-E_{1}\right) \times G \times F \text { for some } n\right) \\
= & 0+\varepsilon \text { (by Step I }) .
\end{aligned}
$$

(Note that $\left\{Z_{n} \in\left(E-E_{1}\right) \times G \times F\right.$ for some $\left.n\right\}$ is contained in $\left\{Z_{1} \in\left(E-E_{1}\right)\right.$ $\times G \times F\}$.)

Since $\varepsilon>0$ is arbitrary, our claim is proven.

Step IV. We have

$$
\begin{aligned}
P_{u_{g}}\left(Z_{n}\right. & \in N \text { for finitely many } n) \\
& =\sum_{k=1}^{\infty} P_{u_{\phi}}\left(Z_{k} \in N, Z_{n} \notin N \text { for all } n \geq k+1\right) \\
& \leq \sum_{k=1}^{\infty} P_{u_{d}}\left(Z_{n} \notin N \text { for all } n \geq k+1\right)=0 .
\end{aligned}
$$

Hence $P_{u_{\sigma}}\left(Z_{n} \in N\right.$ i.o. $)=1$. Q.E.D.

Lemma 3.3. In any semigroup whenever $R \neq \varnothing, R$ is a left ideal of $D$.

It follows from the fact that $P_{x}\left(Z_{n} \in N_{x}\right.$ i.o. $)=1 \Rightarrow P\left(x Z_{n} \in N_{x}\right.$ i.o. $)=1$ which implies $P\left(y x Z_{n} \in y N_{x}\right.$ i.o. $)=1$ and every neighborhood $N_{y x} \supset y N_{x}$ for some $N_{x}$.

Lemma 3.4. If $x \rightarrow y$ i.o. for some $x, y$ then $x D \subset R$.

Proof. By Proposition 3.3, $x D$ is a closed right group. Let $\varepsilon(x D)$ be the set of idempotents of $x D$ and let $f \in \varepsilon(x D)$. Since $y \rightarrow f$, for each neighborhood $N$ of $f$ there is $k$ such that $P_{y}\left(Z_{k} \in N\right)>0$; by lower semicontinuity in $x$ of the function $\mu^{k}\left(x^{-1} N\right)$ (e.g., see [7, p. 143]), there is a neighborhood of $y, N_{y}$, such that $P_{x^{\prime}}\left(Z_{k} \in N\right)>\delta>0$, for all $x^{\prime} \in N_{y}$. Now for $n>k$,

$$
P_{f}\left(Z_{n} \in N\right)=\int P_{f}\left(Z_{n-k} \in d x^{\prime}\right) P_{x^{\prime}}\left(Z_{n-k}^{-1} Z_{n} \in N\right) \geq \delta P_{f}\left(Z_{n-k} \in N_{y}\right) .
$$

By Proposition 3.2, $f \rightarrow y$ i.o., so $\sum P_{f}\left(Z_{n} \in N_{y}\right)=\infty$ by the Borel-Cantelli lemma. Hence by Theorem 3.1 and the above inequality, $f \in R$ for every $f \in \varepsilon(x D)$. Since $x D$ is a right group, $x D=\cup\{x D f$; where $f \in \varepsilon(x D)\}$. Since $R$ is a left ideal of $D$ (Lemma 3.3), we have $x D \subset R$. Q.E.D.

Theorem 3.2. (i) If $x \rightarrow y$ i.o. for some $x, y$, then $R=D=a$ completely simple semigroup and for every pair $z, w \in a D, a=$ any element of $D$, we have $w \rightarrow z$ i.o.

(ii) Either $R=\varnothing$ or $R=D=a$ completely simple subsemigroup. 
Proof. By Proposition 3.3, $D=E \times G \times F$ is completely simple and by Lemma 3.4, there is an idempotent $u_{e f}=\left(e,(f e)^{-1}, f\right) \in R$. Since $\left(e^{\prime}, g, f\right)$ $\left(e,(f e)^{-1}, f\right)=\left(e^{\prime}, g, f\right) \in R$ (since $R$ is a left ideal of $D$ by Lemma 3.3), we obtain $G_{e^{\prime}} \equiv\left\{e^{\prime}\right\} \times G \times\{f\} \subset R$ for all $e^{\prime} \in E$. Since $\left(e,(f e)^{-1}, f\right)\left(e, g, f^{\prime}\right)$ $=\left(e, g, f^{\prime}\right) \subset u_{e f} D \subset R$ by Lemma 3.4, $G_{e f^{\prime}} \subset R$ for every $f^{\prime} \in F$. Consider next $G_{e f^{\prime}}$, for any $e^{\prime} \in E, f^{\prime} \in F$. Since the idempotent $\left(e^{\prime},\left(f e^{\prime}\right)^{-1}, f\right) \in R$, again by the above argument, $G_{e^{\prime} f^{\prime}} \subset R$ and hence $R=D$. The rest of the claims follow easily from Proposition 3.2.(6) Q.E.D.

the left random walk.

Theorem 3.3. $R \neq \varnothing \Leftrightarrow \sum_{n=1}^{\infty} P\left(Z_{n} \in N\right)=\infty$ for all open neighborhoods $N$ of some $x \in D$.

Proof. If $R \neq \varnothing$, then $R=D$ by Theorem 3.2. Hence $\sum P_{y}\left(Z_{n} \in N\right)=\infty$ for all $y \in D \cap N$. Choose $k$ such that $\mu^{k}(D \cap N)>0$. Then

$$
\begin{aligned}
\sum_{n=1}^{\infty} P\left(Z_{n} \in N\right) & \geq \sum_{n=k+1}^{\infty} \int_{D} P_{y}\left(Z_{n-k} \in N\right) \mu^{k}(d y) \\
& =\int \sum_{n=k+1}^{\infty} P_{y}\left(Z_{n-k} \in N\right) \mu^{k}(d y)=\infty .
\end{aligned}
$$

We prove next the " $\Leftarrow$ " part: Let $\sum_{n=1}^{\infty} P\left(Z_{n} \in N\right)=\infty$ for all neighborhoods $N$ of some $x^{\prime} \in D$. Then for each neighborhood $N$ of $x^{\prime}=(e, g, f)$, we have as in the proof of Theorem 3.1, for every positive integer $k$,

$$
P\left(Z_{n} \notin N^{-1} N \text { for all } n \geq k\right)=0 \text {. }
$$

Now given an open neighborhood $N_{1}=N_{0}(e) \times N_{0}\left((f e)^{-1}\right) \times N_{0}(f)$ of $\left(e,(f e)^{-1}\right.$, $f)$, we can find an open neighborhood $N=N_{0}(e) \times g(f e) N_{0}\left((f e)^{-1}\right) \times N_{0}(f)$ of $x^{\prime}=(e, g, f)$ such that $N^{-1} N=N_{1}^{-1} N_{1}$. The reason is as follows: Let $(x, y, z)$ $\in N^{-1} N$. Then there exist $e^{\prime} \in N_{0}(e), g^{\prime} \in N_{0}\left((f e)^{-1}\right), f^{\prime} \in N_{0}(f)$ such that

$$
\left(e^{\prime}, g(f e) g^{\prime}, f^{\prime}\right)(x, y, z) \in N \quad \text { or } \quad\left(e^{\prime}, g(f e) g^{\prime}\left(f^{\prime} x\right) y, z\right) \in N .
$$

This means that $g^{\prime}\left(f^{\prime} x\right) y \in N_{0}\left((f e)^{-1}\right)$ or $\left(e^{\prime}, g^{\prime}, f^{\prime}\right)(x, y, z) \in N_{1}$ or $(x, y, z)$ $\in N_{1}^{-1} N_{1}$. Hence $N^{-1} N \subset N_{1}^{-1} N_{1}$. Similarly $N_{1}^{-1} N_{1} \subset N^{-1} N$. Hence for every positive integer $k, P\left(Z_{n} \notin N_{1}^{-1} N_{1}\right.$ for all $\left.n \geq k\right)=0$, for every open neighborhood $N_{1}$ of $\left(e,(f e)^{-1}, f\right)$. Now by following the proofs of Steps II, III, and IV in Theorem 3.1, we can prove that for every neighborhood $N_{1}$ of $\left(e,(f e)^{-1}, f\right) \equiv u_{e f}$,

$$
P_{u_{f}}\left(Z_{n} \in N_{1} \text { i.o. }\right)=1 \text {. }
$$

Hence $u_{e f} \in R$ and $R \neq \varnothing$. Q.E.D.

(6) If in particular $S=$ a right group and $R \neq \varnothing$, then $R^{\prime}=R=R,=D=$ a right subgroup of $S$ but $R_{\ell}^{\prime}$ may be empty (cf. Example 2.1), where $R_{\ell}^{\prime}, R_{l}$ are the corresponding recurrence sets for 
Corollary 3.1. $R \neq \varnothing \Leftrightarrow \sum P_{x}\left(Z_{n} \in N\right)=\infty$ for all neighborhoods $N$ of some $y \in D, x$ being some fixed element of $D$.

For proof, observe that $\sum P_{x}\left(Z_{n} \in N_{y}\right)=\infty$ for all $N_{y}$ implies that $y$ $\in$ Closure $(x D) \subset\{e\} \times G \times F$, where $x=(e, g, f)$. Also equations (6) and (6') in Theorem 3.1 are valid with $P_{u_{f}}$ replaced by $P_{x}$-measure and the last part of the proof of Theorem 3.3 applies in this case.

Definition 3.3. We say that $Z_{n}$ is a recurrent right random walk if $R \neq \varnothing$.

Theorem 3.4. Suppose that the group factor $G$ is compact in the representation of $S=E \times G \times F$. Then $\left\{Z_{n}\right\}$ is always recurrent and (as in Theorem 3.2) $R=D=$ a completely simple subsemigroup.

Proof. Let $J$ and $H$ be compact subsets of $E$ and $F$ such that $\mu(J \times G \times H)$ $>0$. (This can be done by the regularity of $\mu$.) Let $L=E \times G \times H$ and $L_{1}=J \times G \times H$; then for any $x \in L_{1} \cap D$,

$$
\begin{aligned}
P_{x}\left(Z_{n} \in L_{1} \text { i.o. }\right) & =\lim _{k \rightarrow \infty} P_{x}\left(\cup_{n=k}^{\infty}\left\{Z_{n} \in L\right\}\right) \\
& =\lim _{k \rightarrow \infty} P_{x}\left(\bigcup_{n=k}^{\infty}\left\{X_{n} \in L\right\}\right) \\
& =\lim _{k \rightarrow \infty}\left[1-P_{x}\left(\bigcap_{n=k}^{\infty}\left\{X_{n} \notin L\right\}\right)\right] \\
& =\lim _{k \rightarrow \infty}\left[1-\prod_{n=k}^{\infty} P_{x}\left\{X_{n} \notin L\right\}\right]=1,
\end{aligned}
$$

because $P\left(X_{\text {in }} \notin L\right)=\mu(S-L)<1$, for all $n$. Hence for each subset $J \subset E$ such that $\mu(J \times G \times H)>0$, we have

$$
\begin{aligned}
P\left(Z_{n} \in J \times G \times H \text { i.o. }\right) & =\int_{(E \times G \times F) \cap D} P_{x}\left(Z_{n} \in J \times G \times H \text { i.o. }\right) \mu(d x) \\
& \geq \int_{(J \times G \times H) \cap D} P_{x}\left(Z_{n} \in J \times G \times H \text { i.o. }\right) \mu(d x) \\
& =\mu(J \times G \times H)>0 .
\end{aligned}
$$

Now suppose that $Z_{n}$ is not recurrent. For every $x \in D \cap J \times G \times H$, there exists a neighborhood $N(x)$ such that $\sum P\left(Z_{n} \in N(x)\right)<\infty$, by Theorem 3.3. By the Borel-Cantelli lemma, $P\left(Z_{n} \in N(x)\right.$ i.o. $)=0$. Since $J \times G \times H$ is compact, we can find $x_{1}, x_{2}, \ldots x_{m} \in J \times G \times H$ and neighborhoods $N\left(x_{1}\right)$, $N\left(x_{2}\right) \cdots, N\left(x_{m}\right)$ such that

$$
J \times G \times H \subset \cup_{i=1}^{m} N\left(x_{i}\right)
$$

and

$$
P\left(Z_{n} \in J \times G \times H \text { i.o. }\right) \leq \sum_{k=1}^{m} P\left(Z_{n} \in N\left(x_{k}\right) \text { i.o. }\right)=0
$$


which is a contradiction. Q.E.D.

Theorem 3.5. Let $S=E \times G \times F$. Then $S$ can support a recurrent random walk (i.e., a walk $\left\{Z_{n}\right\}$ with $\left.R \neq \varnothing\right) \Leftrightarrow G$ can support a recurrent random walk.

Proof. The " $\Leftarrow$ " part: It is trivial; just restrict $D$ in $\{e\} \times G \times\{f\}$, and use Definition 3.1 and Theorem 3.2. The " $\Rightarrow$ " part: Let $x=\left(e, g_{x}, f_{x}\right) \in D$ and consider $\{e\} \times G \times F$, Define $U_{8} \equiv\left\{\left(e, g(c e)^{-1}, c\right) ; c \in F\right\}$. Then

$$
\begin{aligned}
U_{g_{1}} U_{g_{2}} & =\left\{\left(e, g_{1}(c e)^{-1}, c\right)\left(e, g_{2}\left(c^{\prime} e\right)^{-1}, c^{\prime}\right) ; c, c^{\prime} \in F\right\} \\
& =\left\{\left(e, g_{1} g_{2}\left(c^{\prime} e\right)^{-1}, c^{\prime}\right) ; c^{\prime} \in F\right\}=U_{g_{182}} .
\end{aligned}
$$

Let $u=\left\{U_{g} ; g \in G\right\}$. It can be shown that $u$ is a group isomorphic to $G$. Recall that $Z_{n}=X_{1} X_{2} \cdots X_{n}$. We define

$$
\begin{array}{ll}
V_{n}=U_{8} & \text { iff } X_{n} \in U_{8} \text { for } n=1,2, \ldots \\
W_{n}=V_{1} V_{2} \cdots V_{n}, & \text { for } n=1,2, \ldots
\end{array}
$$

We note (from the definition) that the $V_{i}$ s are independent because the $X_{i}$ 's are independent. By the group property of the $U_{g}$ 's,

$$
W_{n}=U_{8} \text { if and only if } Z_{n}=X_{1} X_{2} \cdots X_{n} \in U_{8} \text {. }
$$

Hence $\left\{W_{n}\right\}$ is a random walk on $u \cong G .\left(W_{n}=V_{1} V_{2} \cdots V_{n}\right.$ and the $V_{i}$ s are independent.) We note that

(i) $P\left(W_{n} \in E_{A}\right)=P\left(Z_{n} \in \cup E_{A}\right)$, where $A$ is a Borel subset of $G$ and $E_{A}=\left\{U_{g} ; g \in A\right\}, \cup E_{A}=\cup_{g \in A} U_{g}$ and $P\left(W_{n} \in E_{A}\right.$ i.o. $)=P\left(Z_{n} \in \cup E_{A}\right.$ i.o. $)$.

(ii) If $\left\{Z_{n}\right\}$ starts at $x=\left(e, g_{x}, f_{x}\right)$, then $\left\{W_{n}\right\}$ starts at $U_{g_{x}}$.

By (i) and (ii), $\left\{W_{n}\right\}$ is a recurrent right random walk on $\mathcal{U} \cong G$. Q.E.D.

(Note. In the definition of $W_{n}$ above we may define $V_{n}=g$ if $X_{n} \in U_{g}$ since $u$ and $G$ are homeomorphic (given identical topologies); then if $A$ is open in $G, U_{A}$ is open in $\{e\} \times G \times F$ and since $Z_{n}$ starts at $x$, every open set may be restricted in $\{e\} \times G \times F$ and $P_{x}\left(Z_{n} \in U_{A}\right.$ i.o. $)=1$ since $x \in R$. $)$

4. Example 2.2 suggests that when $S$ is a compact semigroup the following result holds.

Lemma 4.1. Let $S$ be a compact semigroup and $D$ the support of the random walks induced by a measure $\mu$. Let $K$ be the kernel of $D$. Then for any neighborhood $U$ of $K$ and any $x \in D$

$$
P\left(Z_{n} \in U \text { i.o. }\right)=P_{x}\left(Z_{n} \in U \text { i.o. }\right)=1 \text {. }
$$


Proof. There exists an open ideal $I$ such that $K \subset I \subset U$ by a result of $\mathrm{R}$. J. Koch and A. D. Wallace [5]. There is $k$ such that $\mu^{k}(I)>0$. We observe that

$$
\mu^{n+k}\left(I^{c}\right)=\int_{I^{c}} \mu^{n}\left(I^{c} x^{-1}\right) \mu^{k}(d x) \leq \mu^{n}\left(I^{c}\right) \mu^{k}\left(I^{c}\right) ; \quad I^{c}=D-I .
$$

Also

$$
\begin{aligned}
\sum \mu^{n}\left(I^{c}\right)= & (\text { Constant })+\mu^{k}\left(I^{c}\right)+\cdots+\mu^{2 k-1}\left(I^{c}\right)+\mu^{2 k}\left(I^{c}\right)+\cdots+\mu^{3 k-1}\left(I^{c}\right) \\
& +\cdots \\
\leq & (\text { Constant })+k \mu^{k}\left(I^{c}\right)+k\left[\mu^{k}\left(I^{c}\right)\right]^{2}+k\left[\mu^{k}\left(I^{c}\right)\right]^{3}+\cdots<\infty .
\end{aligned}
$$

Hence by the Borel-Cantelli lemma, $P\left(Z_{n} \in I^{c}\right.$ i.o. $)=0$ and $P\left(Z_{n} \in I\right.$ i.o. $)$ $=1$. (Observe that also $\sum \mu^{n}\left(I^{c} x^{-1}\right) \leq \sum \mu^{n}\left(I^{c}\right)<\infty$ for every $x \in D$.) Q.E.D.

Theorem 4.1. Let $S$ be a compact abelian semigroup and let $K$ be the kernel of $D$, the support of the random walk $\left\{Z_{n}\right\}$ on $S$. Let $R^{\prime}, R$ be the sets of states which are recurrent in the sense of $(1)$ and Definition 3.1 respectively. Then $R^{\prime}=R=K$.

Proof. Since $S$ is abelian, $K$ is a compact topological group. For any (relatively open) neighborhood $N \subset D$, by restricting our attention to $D$ replacing $S$ by $D$,

$$
P\left(Z_{n} \in N \text { i.o. }\right)=0 \text { or } 1 \text {, }
$$

since $P\left(z_{n} \in D^{c}\right.$ for some $\left.n\right)=0$. (The proof of (8) (known as Hewitt-Savage 0-1 law) can be carried over to locally compact abelian semigroups from [2, p.236]). Since $D$ is compact, it satisfies

(9) $x \notin y D \Rightarrow$ there exist neighborhoods $N_{x}, N_{y}$ such that $N_{y}^{-1} N_{x} \cap D=\varnothing$, i.e., $N_{y} D \cap N_{x}=\varnothing .(7)$

To prove the theorem we first prove:

(10) $a \in R^{\prime} \Rightarrow y \rightarrow a(a \in y D)$ for every $y \in D$.

(11) $a \in R \Rightarrow y \rightarrow a$ (i.e., $a \in y D$ ) for every $y \in D$.

(12) $a \in R^{\prime}$ and $a \rightarrow b \Rightarrow b \in R^{\prime}$.

(13) $x \rightarrow a$ i.o. and $a \rightarrow b \Rightarrow x \rightarrow b$ i.o.

Suppose $a \notin b D$ for some $b \in D$; then by (9) $N_{b}^{-1} N_{a}=\varnothing$ for some $N_{a}$ and some $N_{b}$, so that $N_{b}^{-1} N_{a}^{c}$ is all of $D$. Choosing $k$ such that $P\left(S_{k} \in N_{b}\right)>0$, a contradiction to the recurrence property of $a$ in (10) and (11) will obtain from the following relations respectively:

$$
\begin{aligned}
P\left(Z_{n} \in N_{a}^{c} \text { eventually }\right) & \geq P\left(Z_{n} \in N_{a}^{c} \text { eventually, } Z_{k} \in N_{b}\right) \\
& \geq P\left(Z_{k}^{-1} Z_{n} \in N_{b}^{-1} N_{a}^{c} \text { eventually, } Z_{k} \in N_{b}\right)>0
\end{aligned}
$$

(7) For, if $N_{y} D \cap N_{x} \neq \varnothing$ for all $N_{x}, N_{y}$ then we can find nets $y_{a} \in N_{y}, x_{a} \in N_{x}$ and $d_{a} \in D$ such that $y_{a} d_{a}=x_{a}$ and $y_{a} \rightarrow y, x_{a} \rightarrow x$ which implies $x \in y D$. 
and

$$
\begin{aligned}
P\left(x Z_{k} X_{k+1}\right. & \left.\cdots X_{k+n} \in N_{a}^{c} \text { eventually }\right) \\
& =P\left(Z_{k} x X_{k+1} \cdots X_{k+n} \in N_{a}^{c} \text { eventually }\right)
\end{aligned}
$$

(by the abelian property of $S$ )

$$
\begin{aligned}
& \geq P\left(Z_{k} x X_{k+1} \cdots X_{k+n} \in N_{a}^{c} \text { eventually, } Z_{k} \in N_{b}\right) \\
& \geq P\left(x X_{k+1} \cdots X_{k+n} \in N_{b}^{-1} N_{a}^{c} \text { eventually, } Z_{k} \in N_{b}\right) \\
& >0 .
\end{aligned}
$$

To prove (12) and (13), suppose that $a \rightarrow b$, i.e., $b=a d$ for some $d \in D$. Choose $N_{a}, N_{d}$ such that $N_{a} N_{d} \subset N_{b}$ and choose $k$ such that $P\left(Z_{k} \in N_{d}\right)>0$. Then (12) and (13) follow from the following relations in conjunction with (8) and stationarity:

$$
\begin{aligned}
P\left(Z_{n} \in N_{b} \text { i.o. }\right) & \geq P\left(Z_{n} \in N_{a} N_{d} \text { i.o. }\right) \\
& \geq P\left(Z_{k} \in N_{d}\right) P\left(Z_{k}^{-1} Z_{n} \in N_{a} \text { i.o. }\right)>0
\end{aligned}
$$

and

$P\left(Z_{k} x X_{k+1} \cdots X_{k+n} \in N_{b}\right.$ i.o. $) \geq P\left(Z_{k} \in N_{d}\right) P\left(x X_{k+1} \cdots X_{k+n} \in N_{a}\right.$ i.o. $)>0$.

(Note that $P\left(X_{k+1} \cdots X_{k+n} \in x^{-1} N_{a}\right.$ i.o. $)=P\left(Z_{n} \in x^{-1} N_{a}\right.$ i.o. $)=P\left(x Z_{n}\right.$ $\in N_{a}$ i.o. $)=P_{x}\left(Z_{n} \in N_{a}\right.$ i.o. $)$.)

Next we show that $R^{\prime} \neq \varnothing$ implies $R \neq \varnothing$. Suppose $a \in R^{\prime}=K$. By integration, $P_{x}\left(Z_{n} \in N_{a}\right.$ i.o. $)=1$ for some $x \in D$. Hence for $g \in K, P_{x}\left(Z_{n}\right.$ $\in N_{a}$ i.o. $)=P\left(x Z_{n} \in N_{a}\right.$ i.o. $)=1=P\left(g x Z_{n} \in g N_{a}\right.$ i.o. $)=P_{g x}\left(Z_{n} \in g N_{a}\right.$ i.o. $)=1$. Hence we may assume that $x \rightarrow y$ i.o. for some $x, y \in K$. Hence by using (13), $R \neq \varnothing$.

Next assume $R^{\prime}=\varnothing$. Then for each $x \in K$, there is a neighborhood $N_{x}$ of $x$ such that $P\left(Z_{n} \in N_{x}\right.$ i.o. $)<1$ and hence $P\left(Z_{n} \in N_{x}\right.$ i.o. $)=0$ by (8). Since $K$ is compact, there is a finite cover of $K$ consisting of these $N_{x}$ 's. Say $K \subset U$ $=\bigcup_{i=1}^{n} N_{x}^{(i)}$. Then $P\left(Z_{n} \in U\right.$ i.o. $)=0$, which contradicts Lemma 4.1. Q.E.D.

A remark on invariant measures for $Z_{n}$ when $S$ is completely simple. Suppose $R \neq \varnothing$. Consider any recurrence class of $D$, say $a D, a \in D$. We say that the $\sigma$ finite measure $\nu$ is invariant for $\left\{Z_{n}\right\}$ on $a D$ if it is invariant for the restricted transition function $P(x, A)=\mu\left(x^{-1} A\right)$ on $a D$, i.e., $\int_{a D} P(x, \cdot) \nu(d x)=\nu(\cdot)$. By Theorem 3.2,

(14) For any open $N \subset a D, P_{x}\left(Z_{n} \in N\right.$ i.o. $)=1$ for every $x \in a D$. But (14) is a "Harris recurrence condition" for open sets. By a result of Foguel, condition (14) implies that there is a $\sigma$-finite invariant measure for $Z_{n}$ on $a D$ which is finite on compact sets and positive on open sets. (Actually (14) implies the recurrence 
condition used by Foguel and Horowitz in (1) S. R. Foguel, Existence of $a$ o finite invariant measure for a Markov process on a locally compact space, Israel $\mathbf{J}$. Math. 6 (1968), 1-4; and (2) S. Horowitz, Markov processes on a locally compact space, Israel J. Math. 7 (1969), 311-324; see particularly pp. 312, 317, and 318.)

We finally remark that the referee has pointed out that there are two recent announcements by Jean Larisse in C.R. Acad. Sci. Paris Sér. A 274 (1972), pp. 339-341 and 431-416 which are related to our work, but only deal with discrete semigroups.

\section{REFERENCES}

1. J. F. Berglund and K. H. Hofmann, Compact semitopological semigroups and weakly almost periodic functions, Lecture Notes in Math., no. 42, Springer-Verlag, New York, 1967. MR 36 \#6531.

2. K. L. Chung, A course in probability theory, Harcourt, Brace and World, New York, 1968. MR 37 \#4842.

3. K. L. Chung and W. J. Fuchs, On the distribution of values of sums of random variables, Mem. Amer. Math. Soc. No. 6 (1951). MR 12,722.

4. A. H. Clifford and G. B. Preston, The algebraic theory of semigroups. I,II, Math. Surveys, no. 7, Amer. Math. Soc., Providence, R.I., 1961, 1967. MR 24 \# A2627; MR 36 \# 1558.

5. R. J. Koch and A. D. Wallace, Maximal ideals in compact semigroup, Duke Math. J. 21 (1954), 681-685. MR 16, 112.

6. R. M. Loynes, Products of independent random elements in a topological group, $Z$. Wahrscheinlichkeitstheorie und Verw. Gebiete 1 (1962/63), 446-455. MR 27 \#6293.

7. A. Mukherjea and N. A. Tserpes, Idempotent measures on locally compact semigroups, Proc. Amer. Math. Soc. 29 (1971), 143-151.

8. M. Rosenblatt, Stationary measures for random walks on semigroups, Presented at Sympos. on Semigroups, Wayne State University, Detroit, Mich., 1968, pp. 209-220. MR 41 \#4666.

9.__ Products of independent identically distributed stochastic matrices, J. Math. Anal. Appl. 11 (1965), 1-10.MR 32 \#3098.

10. A. Tortrat, Lois tendues $\mu$ sur une demi-groupe complètement simple $X, Z$. Wahrscheinlichkeitstheorie und Verw. Gebiete 6 (1966), 145-160. MR 35 \# 1063.

11. A. Mukherjea, T. C. Sun and N. A. Tserpes, Random walks on compact semigroups, Proc. Amer. Math. Soc. 39 (1973), 599-605.

Department of Mathematics, Wayne State University, Detroit, Michigan 48202 (Current address of T. C. Sun)

Department of Mathematics, University of South Florida, Tampa, Florida 33620 (Current address of A. Mukherjea and N. A. Tserpes) 